INPLASY

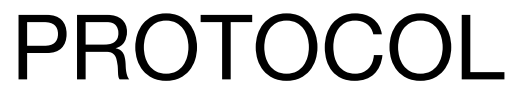

To cite: Ma et al. Efficacy and safety of 225Ac-PSMA-617targeted alpha therapy in metastatic castration-resistant prostate cancer:a systematic review and meta-analysis. Inplasy protocol 202190103. doi:

10.37766/inplasy2021.9.0103

Received: 28 September 2021

Published: 28 September 2021

Corresponding author: Jiao Ma

1109450195@qq.com

Author Affiliation:

Department of Nuclear Medicine, The Affilliated Hospital of Southwest Medical University. Luzhou, Sichuan, PR China

Support: No.

Review Stage at time of this submission: The review has not yet started.

Conflicts of interest:

None declared.

\section{Efficacy and safety of 225Ac- PSMA-617-targeted alpha therapy in metastatic castration-resistant prostate cancer: a systematic review and meta-analysis}

$$
\text { Ma, J1; Li, L2; Liao, T3; Gong, W4; Zhang, C5. }
$$

Review question / Objective: The aim of the meta-analysis is to analysis the efficacy and safety of 225Ac-PSMA-617 in the treatment of metastatic castration-resistant prostate cancer based on existing clinical evidence.

Information sources: We will search articles in two electronic database including PubMed and EMBASE.

Main outcome(s): Biochemical response (any degree of PSA decline and PSA decline $>50 \%$ ) The biochemical response is evaluated according to the criteria defined by the Prostate Cancer Clinical Trials Working Group 3 (PCWG3).Patients whose PSA decreased by $\geq 50 \%$ from baseline were defined as a biochemically significant response.

INPLASY registration number: This protocol was registered with the International Platform of Registered Systematic Review and Meta-Analysis Protocols (INPLASY) on 27 September 2021 and was last updated on 27 September 2021 (registration number INPLASY202190103).

\section{INTRODUCTION}

Review question / Objective: The aim of the meta-analysis is to analysis the efficacy and safety of 225Ac-PSMA-617 in the treatment of metastatic castration- resistant prostate cancer based on existing clinical evidence.

Condition being studied: Prostate cancer is one of the most common malignant tumors in men worldwide. According to the latest report of global cancer statistics in 2020, 
the incidence and mortality of prostate cancer rank the 2nd and 5th among male malignancies in the world.At present, the main treatment methods for prostate cancer include radical surgical resection, radiotherapy, chemotherapy, local treatment, and androgen deprivation therapy. As the disease progresses, the efficacy of these therapies will gradually decrease or even be completely ineffective. In recent years, radionuclide-labeled prostate-specific membrane antigen ligands have been used in the diagnosis and treatment of prostate cancer, and have achieved good results. 177Lu-PSMA-617 has shown good effectiveness, safety and easy availability for MCRPC. However, most patients still tolerate 177Lu treatment or their condition continues to progress after treatment. Compared with 177Lu, 225Ac ray has higher energy, shorter range, and stronger killing effect on tumor cells. In addition, 225Ac-PSMA-617 also has the advantage of targeting any metastatic tissue, and it has a good application prospect for small tumors, scattered cancers and micrometastasis. However, there are few systematic reviews or metaanalysis studies on the efficacy and safety of 225Ac-PSMA-617 targeted therapy for MCRPC in the published literature.

\section{METHODS}

Search strategy: We will search articles in two electronic database including PubMed and EMBASE. All the English publications until 30 July 2021 will be searched without any restriction of countries. The searching string will be built as follows:\{(prostate* neoplasm* [MeSH] OR prostate cancer)AND (Actinium-225 [MeSH] OR 225Ac OR 225Actinium OR Ac-225)\}.

Participant or population: Inclusion criteria:No less than 10 patients who have been diagnosed as MCRPC by $68 \mathrm{Ga}-$ PSMA-11 PET/CT, Completed at least 1 cycle of 225Ac-PSMA-617 treatment. Exclusion criteria:mCRPC patients with severe leukopenia, low platelets, renal failure, and end-stage tumors who cannot tolerate 225Ac-PSMA-617 treatment.
Intervention: Completed at least 1 cycle of 225Ac-PSMA-617 treatment.

Comparator: No.

Study designs to be included: The retrospective study will be included.

Eligibility criteria: No less than 10 patients who have been diagnosed as MCRPC by 68Ga-PSMA-11 PET/CT, Completed at least 1 cycle of 225Ac-PSMA-617 treatment.All the 97English publications until 31 July 2021 will be searched without any restriction of countries.

Information sources: We will search articles in two electronic database including PubMed and EMBASE.

Main outcome(s): Biochemical response (any degree of PSA decline and PSA decline $>50 \%$ ) The biochemical response is evaluated according to the criteria defined by the Prostate Cancer Clinical Trials Working Group 3 (PCWG3).Patients whose PSA decreased by $\geq 50 \%$ from baseline were defined as a biochemically significant response.

Additional outcome(s): Overall survival(OS), Progression-free survival(PFS), Molecular response, Reatment-related toxicity.

Quality assessment / Risk of bias analysis: Use the Newcastle-Ottawascale (NOS) scale to assess the methodological quality of the literature, the quality of the literature with NOS $\geq 6$ points is high quality. Perform publication bias analysis on biochemical reactions. Qualitative and quantitative evaluation uses the funnel chart and egger test method in stata 16.0.

Strategy of data synthesis: Meta-analysis of biochemical reactions, overall survival (OS), progression-free survival (PFS), molecular reactions and treatment-related toxic reactions was performed using STATA16.0, and a forest plot was drawn for the outcomes. $I^{2}$ statistic is used for heterogeneity testing. If there is no 
significant heterogeneity between studies $\left(I^{2} \leq 50 \%, P<0.10\right)$, the fixed effects model is used to merge the data; if there is significant heterogeneity between studies $\left(I^{2}>50 \%, P \geq 0.10\right)$, the data was merged using a random effects model.

Subgroup analysis: No.

Sensitivity analysis: No.

Country(ies) involved: China.

Keywords: 225Ac-PSMA-617; metastatic castration-resistant prostate cancer; a nuclide therapy; meta-analysis.

Contributions of each author:

Author 1 - Jiao Ma.

Author 2 - Lanyin Li.

Author 3 - Taiping Liao.

Author 4 - Weidong Gong.

Author 5 - Chunyin Zhang. 